journal club

\title{
Bei chronischer Depression ähnlich effektiv wie Escitalopram
}

Fragestellung: Non-Inferiority-Studie mit Vergleich von 22 Sitzungen CABSP und $20 \mathrm{mg}$ Escitalopram über 28 Wochen.

Hintergrund: Die Behandlung einer persistierenden „chronischen“ Depression ist eine große Herausforderung. Die Remissionsraten in den meisten Studien liegen deutlich unter $40 \%$. Es ist unklar, welche psycho- oder pharmakotherapeutischen Methoden einen wesentlichen Unterschied zum Spontanverlauf der Erkrankung ergeben. CBASP ist die erste spezifisch auf bei chronischer Depression beobachtete Fertigkeitendefizite ausgerichtete Psychotherapie.

Schramm E, Zobel I, Schoepf D et al. Cognitive Behavioral Analysis System of Psychotherapy versus escitalopram in chronic major depression. Psychother Psychosom 2015; 84: 227-40
Patienten und Methodik: 60 Patienten mit chronischer Depression wurden randomisiert zwei Studienarmen zugeteilt: 1) 22 Sitzungen CBASP plus klinisches Management; 2) Pharmakotherapie mit Es- citalopram mit einer Dosierung von initial $10 \mathrm{mg}$ und Steigerung auf $20 \mathrm{mg}$ plus klinisches Management. Bei einer ausbleibenden Verbesserung nach acht Wochen (Rückgang der Punktzahl im MADRS weniger als 20\%) wurde jeweils zusätzlich Escitalopram beziehungsweise CBASP zu der Behandlung hinzugefügt. Der primäre Endpunkt war die Punktzahl des MADRS Score.

Ergebnisse: Zu Therapiebeginn bestand eine hohe Depressionsschwere von 25 bis 30 MADRS-Punkten, die nach 28 Wochen Beobachtung auf 8 bis 20 Punkte reduziert war. Es gab keinen Haupteffekt des Behandlungsarms. Die Remissionsraten waren $37 \%$ in der Gruppe mit CBASP-Monotherapie, 50\% in der Gruppe mit Escitalopram-Monotherapie und $30 \%$ in der Gruppe mit der Augmentation durch die jeweils andere Methode.

Schlussfolgerungen: Beide Behandlungsmethoden zeigen mittlere Effektstärken bezüglich der Symptomreduktion der Depression. Die Remissionsraten, das primäre Outcome-Kriterium sind aus statistischer Perspektive ähnlich.

\section{- Kommentar von Ulrich Schweiger, Lübeck}

\section{Der Status von CBASP - Hoffnungen und Unsicherheiten}

CBASP ist eine wichtige Fortentwicklung innerhalb der Verhaltenstherapie und die erste spezifisch auf chronische Depression ausgerichtete Methode. Der von CBASP gewählte Ansatzpunkt an sozial-kognitiven Prozessen ist herausfordernd, faszinierend und für die psychotherapeutische Community mit der Hoffnung verbunden, bei dieser Zielgruppe endlich höhere Erfolgsraten zu haben. Gleichzeitig gibt es einen erschreckenden Mangel an qualitativ hochwertigen Studien, die die Wirksamkeit von CBASP untersuchen. Bei der Studie von Schramm et al. handelt sich um die fünfte publizierte randomisierte Studie zu CBASP. Es ist die dritte Studie, die einen Vergleich zu Pharmakotherapie beinhaltet. Ähnlich wie bei den Vorstudien ergibt sich kurz- und mittelfristig ein ähnlicher Verlauf der krankheitsdefinierenden Symptome bei Pharmakotherapie und CBASP. Die mittleren bis hohen Effektstärken von CBASP geben Hoffnung, eine effektive Behandlung zu haben. Gleichzeitig beantwortet die Studie wesentliche Fragen nicht. Sie kann aufgrund der Zahl von initial eingeschlossenen $60 \mathrm{~Pa}$ tienten, deren Outcome aufgrund des Crossover am Ende drei Armen zuzuordnen sind, nur als Pilotstudie betrachtet werden. Klinisch relevante Überlegenheit oder Unterlegenheit kann bei dieser Studiengröße weder erfasst noch ausgeschlossen werden. Die wichtigere unbeantwortete Frage ist, wie sich die Therapiemethoden langfristig auswirken. Remission innerhalb von 28 Wochen und die Punktzahl im MADRS sind problematische Endpunkte. Es besteht eine hohe Konvergenz aller pharmako- logischen und psychologischen Methoden der Depressionsbehandlung bezüglich kurz- und mittelfristiger symptombezogener Outcomes, langfristig hat Psychotherapie möglicherweise die bessere Nachhaltigkeit [1]. CBASP ist unter anderem ein Fertigkeitentraining. Verbesserte sozial-kognitive Fertigkeiten ergeben möglicherweise einen besseren Langzeitverlauf als pharmakologische Behandlungen. Nicht ohne Grund haben deshalb andere Studien auch diese sekundären Endpunkte untersucht $[2,3]$.
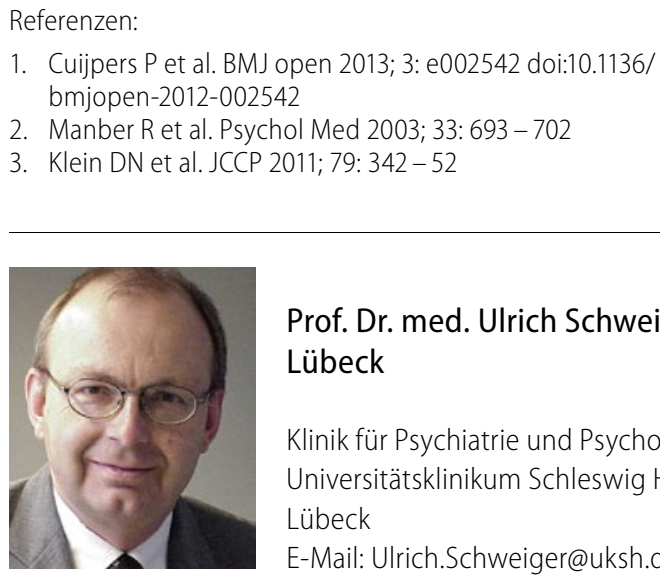

Prof. Dr. med. Ulrich Schweiger, Lübeck

Klinik für Psychiatrie und Psychotherapie, Universitätsklinikum Schleswig Holstein, Lübeck

E-Mail: Ulrich.Schweiger@uksh.de 